Peer-to- Peer cont ent s del i very syst em consi dering net wor $k$ di st ance

\begin{tabular}{|l|l|}
\hline 著者 & Okubo Takuya, Ueda Kazunor i \\
\hline $\begin{array}{l}\text { j our nal or } \\
\text { publ i cat i on t i t l e }\end{array}$ & $\begin{array}{l}\text { Net work Oper at i ons and Nanagenent Symposi um } \\
\text { ( APNOMS), 2011 13t h Asi a Paci f i c }\end{array}$ \\
\hline page range & 1- 4 \\
\hline year & 2011- 09 \\
\hline URL & ht t p: //hdl . handl e. net /10173/976 \\
\hline
\end{tabular}




\section{Peer-to-Peer contents delivery system considering network distance}

\author{
Takuya Okubo \\ Kochi University of Technology \\ Kochi, Japan
}

\author{
Kazunori Ueda \\ Kochi University of Technology \\ Kochi, Japan
}

\begin{abstract}
Network traffic on the Internet has been increasing in recent years. Some reports by government institutions indicate that Peer-to-Peer (P2P) traffic consumes 30\% of network bandwidth on the Internet. In most current P2P file-sharing software, computers, which are called "peer" get files not only from computers that are nearby on the network but also from those that are far away. This is because the computers do not consider the relative location of other computers on the Internet. We regard Round Trip Time (RTT) or Time To Live (TTL) as the network distance and design a new P2P content delivery system. In this system, to decrease waste, peers on the P2P file-sharing network prioritize nearby peers when obtaining files. Peers search for other peers that have desired files using clusters, based on network distance and trend of contents which a peer has. Peers search for popular files from other peers in distance-based clusters and search for less popular files from peers in trendbased clusters. Simulation results indicate our system facilitates a reduction in traffic in the whole network.

Index Terms-Peer-to-Peer, File sharing.
\end{abstract}

\section{INTRODUCTION}

P2P file-sharing software does not require a server, so such software can be managed easily and it is inexpensive. Therefore, many researchers have conducted studies on P2P file-sharing systems. In 1999, Napster[1] was released as file-sharing software for music. It was the pioneer of P2P file-sharing software. It ceased service in 2000 because of legal action taken by the Recording Industry Association of America. Gnutella[2] was adopted as a pure P2P system that does not require a server. Therefore, the Gnutella network can withstand strong failure. KaZaA[3] did not adopt a server; rather, it used supernodes that work like a server. BitTorrent[4] is a P2P file transfer protocol and software application. In BitTorrent, the user sends a part of the file. These parts are combined from many uploading users so that the user gets the complete files. This approach allows low-bandwidth users to join in files distribution.

Usage trends of the Internet show that in recent years, network traffic on the Internet has been increasing. Download traffic has consume bandwidth about 990 Gbps in Japan since 2008 according to a press release of the Ministry of Internal Affairs and Communications[5]. This download traffic is 1.2 times that in 2007. About $30 \%$ of all upstream bandwidth is P2P bandwidth[6]. That is, traffic of current P2P file-sharing software consumes $30 \%$ of all network bandwidth.

As network bandwidth disappears, ISPs can either upgrade network equipment or improves transfer speeds. However, this is expensive. Therefore, ISPs are instead executing bandwidth control.

We propose to decrease $\mathrm{P} 2 \mathrm{P}$ traffic by considering the network distance. We consider the network distance in the P2P content delivery system. This avoids wasteful traffic. We construct a $\mathrm{P} 2 \mathrm{P}$ content delivery system that considers the network distance using a P2P clustering algorithm[7]. A downloading user gets content from a peer in their nearby network. Therefore, P2P traffic decreases.

\section{P2P Network MOdeL}

The P2P network model requires no server management or maintenance because it has no servers. Therefore, the P2P network model is not as expensive as a client-server model. Further, each peer in the P2P network model directly connects to another peer and distributes files on the network, so the load is distributed across each peer. (Fig.1)

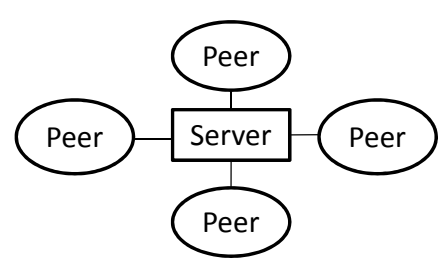

Client-server model

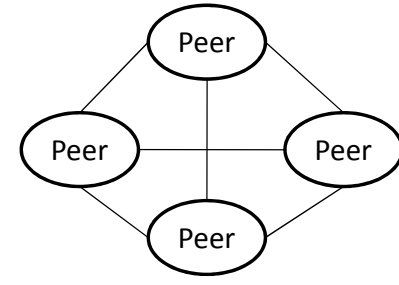

P2P model
Fig. 1. Network model

\section{A. Current P2P File-Sharing Software}

Current P2P file-sharing software includes Freenet[8], Gnutella[2], BitTorrent[4], Winny[9].

1) Freenet: Freenet [8] is free software that advocates freedom of speech. Therefore, it is designed for high anonymity. However, as a result, it sacrifices on transfer speed and convenience. This high anonymity cannot be cracked by the power of the state, and Freenet users cannot conduct searches based on file names.

2) Gnutella: Gnutella[2] is a P2P protocol and file-sharing client. Gnutella has no servers and runs only between peers. Therefore, the Gnutella network can withstand strong failure. 
3) Winny: Winny[9] is P2P file-sharing software that was made in Japan. Winny's network structure is constructed as a hierarchical cluster, based on speeds and keywords that the user sets. If the user opens files, a key is created for each file, and the key is distributed to nearby peers. The key contains metadata. The user gets files using the key because the key indicates the location of the file.

4) BitTorrent: BitTorrent[4] is a P2P-file sharing protocol and software application. Files in BitTorrent are divided into pieces and tracked using torrent files. A searching peer first gets a torrent file for a web server. The torrent file contains metadata about the tracker. The tracker is a server that supplies the IP addresses of the various peers in the network to the searcher. The searching user begins downloading the supplied piece of the file. The downloading peer simultaneously offers other pieces of the file for download by others. In this way, the number of file-up-loading peers increases, supplying a complete file.

\section{B. $P 4 P$}

$\mathrm{P} 4 \mathrm{P}$ is a file transfer protocol for $\mathrm{P} 2 \mathrm{P}$. The $\mathrm{P} 4 \mathrm{P}$ working group of the Distributed Computing Industry Association[10] maintains P4P. P4P stands for Proactive network Provider Participation for P2P or Provider Portal for P2P. An ISP provides information on the constructed network, available bandwidth, packet loss rate, etc., to P2P applications so that $\mathrm{P} 2 \mathrm{P}$ applications can manage the network more efficiently (Fig.2). P4P manages traffic according to this information, improving network efficiency and transfer speeds.

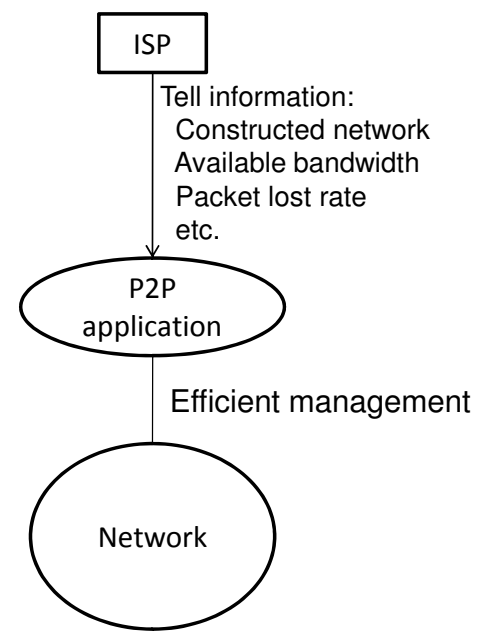

Fig. 2. P4P: ISP provides information to $\mathrm{P} 2 \mathrm{P}$ application for efficient network management.

\section{P2P Clustering Algorithm}

A P2P clustering algorithm was also proposed[7]. A peer executes clustering automatically and hierarchically. Peers near to one another on the network belong to the same cluster. Peers that are far from one another in the network belong to another cluster. Further, peers belong to hierarchical clusters; the upper-level cluster contains peers that are farther away than those in the lower-level cluster. Each peer has a pointer to all of the peers in the lowest-level cluster, and in each rank cluster, the peer has a pointer to one of the other peers in the same upper cluster and all the clusters that are not joined.

Fig.3 depicts an example of the pointers to each peer. Here, peer A has pointers. Peer A belongs to $\langle\mathrm{C} 0\rangle$ in the top-layer cluster (layer 1), so Peer A has a pointer to another peer (Peer G) in $\langle\mathrm{C} 1\rangle$. Peer A belongs to $\langle\mathrm{C} 00\rangle$ in the bottom-layer cluster (layer 2), so Peer A has pointers to another peer (Peer C) in $\langle\mathrm{CO} 1\rangle$ and to all of its peers (Peer B) in the same bottom-layer cluster $(<\mathrm{CO} 0\rangle)$.

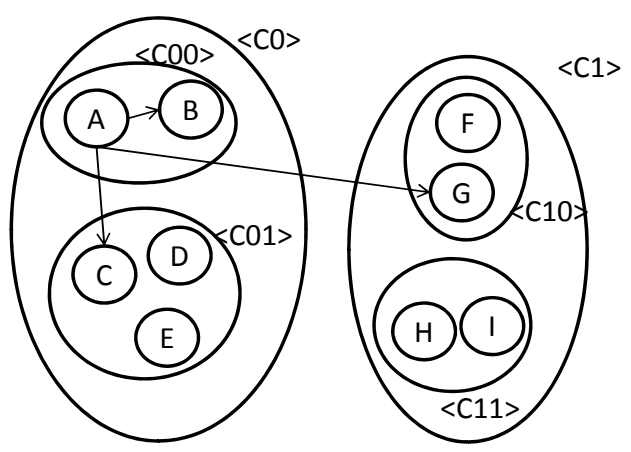

Fig. 3. Example of pointer to each peer

Each peer has a cluster table that is uses to manage the above information. TABLEI is an example of a cluster table. In TABLEI, the number of layers is $d$ and the number of subcluster is $\mathrm{n}$. The information on clusters pertains to either joined clusters or clusters that have not been joined. The information on clusters is listed in TABLEII. The cluster Id is an identifier for each cluster. The Faraway Peers List arranges peers based on their distance in the network. If a Delegate Peer breaks down, one of the Backup Peers becomes the Delegate Peer.

TABLE I

Cluster TABLE (NUMBER OF LAYERS IS D AND NUMBER OF SUB CLUSTESRS IS N)

\begin{tabular}{c|c|c|c|c|c}
\hline layer & $\begin{array}{c}\text { joined } \\
\text { cluster }\end{array}$ & $\begin{array}{c}\text { another } \\
\text { cluster 1 }\end{array}$ & $\begin{array}{c}\text { another } \\
\text { cluster 2 }\end{array}$ & $\ldots$ & $\begin{array}{c}\text { another } \\
\text { cluster n-1 }\end{array}$ \\
\hline 1 & $<\mathrm{C} 0>$ & $<\mathrm{C} 1>$ & $<\mathrm{C} 2>$ & $\ldots$ & $<\mathrm{Cn}>$ \\
\hline 2 & $<\mathrm{C} 00>$ & $<\mathrm{C} 01>$ & $<\mathrm{C} 02>$ & $\ldots$ & $<\mathrm{C} 0 \mathrm{n}\rangle$ \\
\hline$\ldots$ & $\ldots$ & $\ldots$ & $\ldots$ & $\ldots$ & $\ldots$ \\
\hline $\mathrm{d}$ & $\langle\mathrm{Cd} 0\rangle$ & $<\mathrm{Cd} 1>$ & $<\mathrm{Cd} 2\rangle$ & $\ldots$ & $<\mathrm{Cdn}>$ \\
\hline
\end{tabular}

When a new peer joins the network, the clustering processes are executed. If the number of subclusters is fewer than the threshold, the peer creates a new cluster. If the number of subclusters is not fewer than the threshold, the peer joins a cluster in the nearby network. The peer repeats this process, proceeding up to the top layer, until finds the desired content. 
TABLE II

CLUSTER INFORMATION

\begin{tabular}{c|c|c}
\hline \multicolumn{2}{c|}{ which cluster } & information \\
\hline \multirow{3}{*}{ joined cluster } & not bottom & $\begin{array}{c}\text { Cluster Id, } \\
\text { Faraway Peers List, } \\
\text { Delegate Peer (myself) }\end{array}$ \\
\cline { 2 - 3 } & \multirow{2}{*}{ bottom } & $\begin{array}{c}\text { Cluster Id, All of Peers List, } \\
\text { Faraway Peers List, } \\
\text { Delegate Peer (myself) }\end{array}$ \\
\hline \multirow{2}{*}{ another cluster } & $\begin{array}{c}\text { Cluster Id, } \\
\text { Delegate Peer, } \\
\text { Backup Peers List }\end{array}$ \\
\hline
\end{tabular}

The appropriate number of subclusters is 2 . The appropriate depth is $\left(\log _{2} n-1\right) \times 2$ ( $\mathrm{n}$ is number of peer).

\section{OUTLINE}

We propose to decrease P2P traffic on P2P content delivery systems by considering the network distance. We construct a P2P content delivery system that considers the network distance using a P2P clustering algorithm. Therefore, peers get files from their nearby network, rather than peers that are far away. P2P traffic is expected to decrease as a result. We regard RTT or TTL as the network distance.

For example, if we treat the TTL as the network distance. Peer B and Peer C have the same content and Peer A searches for this content. The network distance is 2 hops from Peer A to Peer B. The network distance is 3 hops from Peer A to Peer C. Peer B is nearer to Peer A than Peer C. If Peer A can gets the file from Peer B, it decreases the required network traffic (Fig.4.).

The P2P content delivery system we use in our research assumes that content will be redistributed. Therefore, content will be supplied in response to many requests from many holder peers and many distributed peers. If the peer wishes to download content that has many holder peers, the peer searches for the content based on the network distance. This is because many peers, including peers in the nearby network, will have popular content, which is heavily requested. If the peer wishes to download content that is held by only a few peers, the searcher attempts to find it based on the contentby-content trend of the current P2P file-sharing software application.

A peer switches between constructed clusters based on the content's popularity rating when it conducts its searches. Clusters are either distance-based or trend-based (Fig.4). A distance-based cluster is a cluster that is formed based on the network distance. A trend-based cluster is a cluster that is based on content trends. The popularity rating is the number of peers that have the content. A peer records the number of request it has received because this indicates the popularity rating of the content. The peer then decides to use a distancebased cluster or trend-based cluster on the obtained popularity rating.
The content and peers in our system have multiple elements. The elements express kind of content or peer. The elements are used when a peer searches for content and joins a trend-based cluster. The peer element is determined by whether content is held.

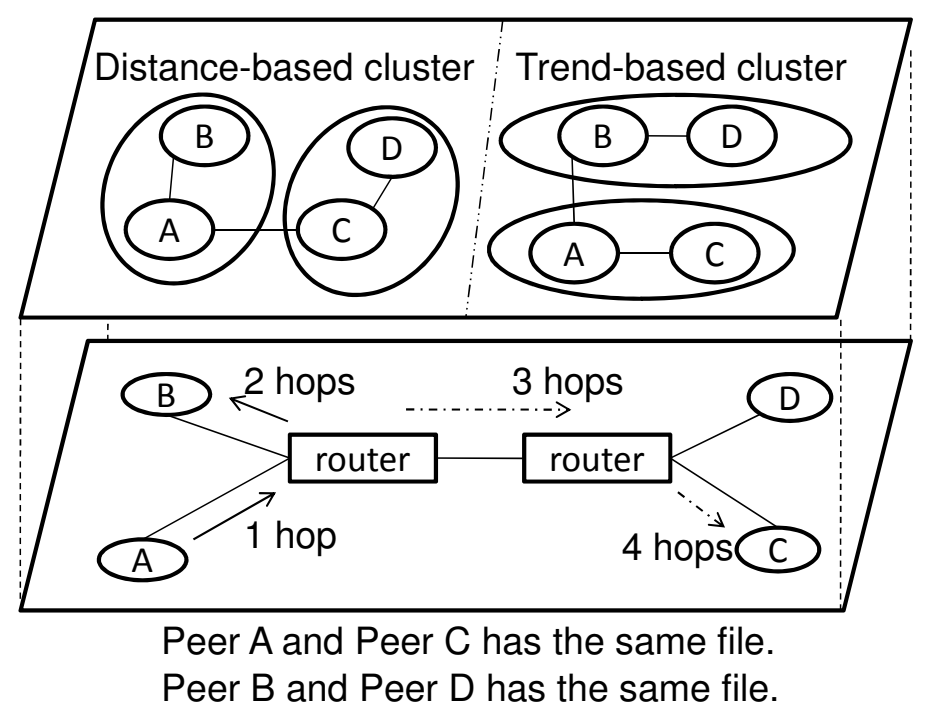

Fig. 4. Decrease in network traffic by considering network distance

\section{A. Content Search using Distance-based Cluster}

The content search procedure using a distance-based cluster is as follows. First, the peer sends a request for the held content list that indicates the desired element to other peers in the same bottom-layer cluster. The receiving peer then sends its held content list to the peer that sent the request. Next, if the received content list includes the desired content, the searching peer retrieves the content and the process completes. If the received content list does not include the desired content, the peer sends a request for a held content list that indicates the desired element to the delegate peer one layer above the bottom-layer cluster. The receiving peer then sends its held content list to the searching peer. It then sends the request to the delegate peer of a subcluster and sends the request to all peers in the same bottom cluster. Then, the receiving peers send their held content list to the searching peer. The peer then checks whether the received content lists have the desired content. The peer then repeats this process, proceeding up to the top layer, until finds the desired content.

Peers in our system begin searching for content with peers in the nearby network. Therefore, the peer prioritized nearby peers when searching for content.

\section{Simulation}

We performed a simulation to measure the network distance. We implemented the base of the system on PIAX (version 2.1)[11], which is a $\mathrm{P} 2 \mathrm{P}$ framework. We implemented the $\mathrm{P} 2 \mathrm{P}$ clustering algorithm and content search algorithm. 


\section{A. Simulation}

We implemented the P2P clustering algorithm and search algorithm on PIAX. We ran the simulation under the following conditions. The number of peers was 100. The distance-based cluster had a depth of 10 . The number of subclusters in the distance-based cluster was 2 . The number of contents files was 63. Each peer had some content. The network distance was based on the TTL (TABLEIII).

TABLE III

SIMULATION PARAMETERS

\begin{tabular}{c|c}
\hline Number of peers & 100 \\
\hline Depth of distance-based cluster & 10 \\
\hline Number of subclusters & 2 \\
\hline Number of contents & 63 \\
\hline Network distance & TTL \\
\hline
\end{tabular}

We performed a simulation that consisted of the following steps. First, 100 peers joined the network and clusters. The peers then waited until all peers had finished rejoining. Rejoining is when peers change cluster membership to produce optimal clustering. Each peer then searched the content. We then measured the network distance between the holding peer and searching peer. The steps in this procedure are detailed below.

\section{B. Simulation Result}

Fig.5 presents the simulation results. The horizontal axis represents the popularity rating of the content. The vertical axis represents the network distance to the holding peer. As it evident, the distance-based cluster is almost equal to the Trend-based cluster in terms of the network distance.

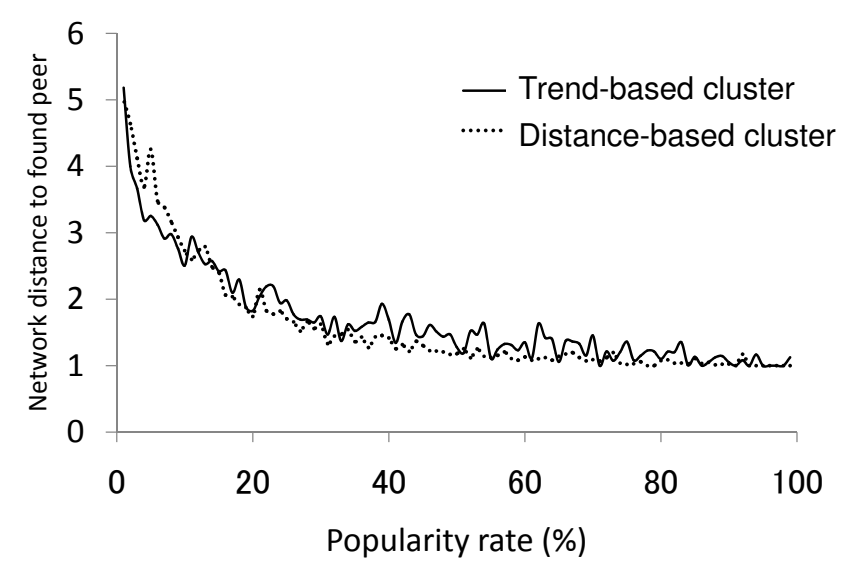

Fig. 5. Simulation results

\section{Discussion}

The simulation produced almost the same results, regardless of whether a distance-based cluster or trend-based cluster. However, the distance-based cluster produced network distances that were nearer than those produced by the trendbased cluster, which is based on popularity rating. The trendbased cluster produced network distances nearer than those of the distance-based cluster when the popularity rating of the content was low. Therefore, if the peer searches for content that has a high popularity rating, the search is carried out using a distance-based cluster. If the peer searches for content that has a low popularity rating, trend-based cluster is used. The peer will then get the content from a peer that is nearby in the network, thus decreasing network traffic.

We have performed these simulations for other conditions and parameters as well.

\section{CONClusion}

Network traffic on the Internet has been increasing in recent years. About $30 \%$ of network bandwidth is consumed by $\mathrm{P} 2 \mathrm{P}$ file sharing. Therefore, the purpose of this research is to decrease P2P traffic by considering the network distance. We regard the TTL or RTT as the network distance. Network traffic can be reduced if a peer gets content from another peer that is nearby in the network. In our system, a peer switches between clusters based on the popularity ratings of the content they seek. Clusters are distance-based cluster and trend-based cluster.

The simulation produced almost the same result, regardless of whether a distance-based cluster or trend-based cluster was used. However, there was a slight difference; when the content's popularity rating was high, the use of the distancebased cluster resulted in peers downloading content from other peers that were closer to themselves on the network than when the trend-based cluster was used. Therefore, we believe that if the peer searches for content that has a high popularity rating, the search would be optimal if carried out using a distancebased cluster. Network traffic will then be decreased.

\section{REFERENCES}

[1] N. LLC, "Napster," available at http:///www.napster.com/, (accessed 2011-05-13).

[2] G. Kan, Chapter 8 in the book PEER-TO-PEER: Harnessing the Power of Disrutive Technologies, A. Oram, Ed. O'REILLY, 2001.

[3] Kazaa, "Kazaa," available at http://www.kazaa.com, (accessed 2011-0513).

[4] BitTorrent, Inc, "Bittorrent," available at http://www.bittorrent.com/, (accessed 2010-02-04).

[5] MIC, "MIC's press release," available at http://www.soumu.go.jp/menu_ news/s-news/090227_3.html, (accessed 2010-02-02).

[6] Sandvine, "2009 global broadband phenomena," 2009.

[7] T. Ueda, K. Abe, H. Ishibashi, and T. Matsuura, "Decentralized hierarchical internet hosts clustering (network system management technolo-

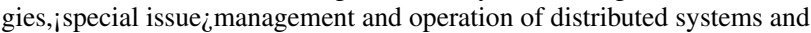
the internet)," Information Processing Society of Japan, vol. 47, no. 4, pp. 1063-1076, 2006.

[8] I. Clarke, O. Sandberg, B. Wiley, and T. W. Hong, "Freenet: A distributed anonymous information storage and retrieval system," in Proceedings of the International Workshop on Design Issues in Anonymity and Unobservability, Jul. 2000, pp. 46-66.

[9] I. Kaneko, The Technology of Winny. ASCII, 2005.

[10] H. Xie, Y. R. Yang, A. Krishnamurthy, Y. Liu, and A. Silberschatz, "P4P: Provider portal for applications," in ACM SIGCOMM Computer Communication Review, vol. 38, no. 4, Seattle, USA, Oct. 2008, pp. 351-362.

[11] B. Inc and O. University, "PIAX," available at http://www.piax.org/, (accessed 2010-02-04). 